\title{
Counting the neurological cost of COVID-19
}

\section{Abdul Mannan Baig}

The neurological deficits caused by COVID-19, which were first reported in the early months of 2020, continue to intrigue neurologists and health-care professionals worldwide. As two new studies highlight, these manifestations are frequent and are expected to increase the burden of morbidity and mortality in the acute and chronic phases of COVID-19.

Refers to Misra, S. et al. Frequency of neurologic manifestations in COVID-19: a systematic review and meta-analysis. Neurology https://doi.org/10.1212/WNL.0000000000012930 (2021) | Valencia Sanchez, C. et al. Autoimmune encephalitis post-SARS-CoV-2 infection: case frequency, findings, and outcomes. Neurology https:// doi.org/10.1212/WNL.0000000000012931 (2021).

The ongoing global COVID-19 pandemic, caused by the SARS-CoV-2 virus, has affected individuals from all walks of life, imposing a colossal burden on clinicians and overwhelming the health-care apparatus in many countries. Despite multiple reports of neurological deficits in patients with COVID-19 from across the world, the precise incidence of these manifestations has remained unknown, and two new studies have attempted to address this issue.

In a systematic review and meta-analysis ${ }^{1}$, Shubham Misra and colleagues detailed the occurrence of syndromic expression of neurological involvement in COVID-19 and probed the association of neurological indices with disease severity and mortality in patients with COVID-19. By analysing data from 55 countries, they identified 41 neurological manifestations that can occur in conjunction with COVID-19. Up to one-third of individuals with COVID-19 were estimated to exhibit at least one of these manifestations.

\section{SARS-CoV-2 was recognized} as a potential neurotropic virus in the early months of 2020

Differences in the prevalence of the various neurological symptoms were observed between older (aged 60 years or more) and young (aged less than 18 years) subpopulations. Acute confusion or delirium, fatigue and myalgia tended to dominate in the older group. In patients aged under 18 years, the most common neurological symptoms were impairment of smell and/or taste and headache, and seizures were also noted in some individuals. Alarmingly, mortality in patients with COVID-19 who had one or more neurological manifestations was found to be $27 \%$ and was especially high in patients aged 60 years or more. The finding that the prevalence of stroke was $1.2 \%$ in people with COVID-19, compared with only $0.2 \%$ in those with influenza, is also concerning ${ }^{1}$.

In the second study, Cristina Valencia Sanchez et al. ${ }^{2}$ evaluated the frequency of antibodies against the SARS-CoV-2 spike protein in serum samples from 556 consecutive patients who had undergone neural antibody testing as part of the diagnostic evaluation for autoimmune encephalitis. In this cohort, 18 (3\%) of the patients tested positive for antibodies against SARS-CoV-2. Overall, the number of individuals with post-COVID-19 autoimmune encephalitis encountered by the researchers in their clinic was low, representing only $0.05 \%$ of patients with COVID19-related diagnoses. In the few patients who developed autoimmune encephalitis following SARS-CoV-2 infection, neurological manifestations included seizures, mood disturbances, delirium and gait ataxia, sometimes accompanied by abnormal findings on brain MRI.

From a prophylactic and therapeutic perspective, the neurological manifestations of COVID-19 raise several questions. First, do the observed neurological deficits truly reflect neuronal injury? Second, what mechanisms are involved in neuronal damage in COVID-19? Third, will the disabilities caused by neurological deficits continue to progress if COVID-19 takes a protracted course? Last, are any means available to reduce the incidence of neurological involvement in COVID-19?
Skeletal muscle injury, fatigue, weakness, headaches and inability to concentrate are some of the many clinical complaints in patients with COVID-19 that could be of non-neural origin ${ }^{1}$. Therefore, more emphasis should probably be placed on clinical features such as recent persistent cognitive changes involving memory and reasoning after a COVID-19 diagnosis, seizures that cannot be explained by other causes, cranial nerve palsies, delirium, smell and taste impairment, impaired consciousness and gait ataxia ${ }^{1,2}$, with associated findings on imaging that suggest a genuine neurological deficit.

Although COVID-19 was initially thought to be a disease of the respiratory passages, SARS-CoV-2 was recognized as a potential neurotropic virus in the early months of 2020, and the possibility that the virus could access the brain via the olfactory mucosa and the cribriform plate was raised ${ }^{3}$. Subsequent studies have reported involvement of the olfactory pathways in the spread of SARS-CoV-2 to the brain ${ }^{4,5}$. The neurotropism of SARS-CoV-2 could be mediated by angiotensin-converting enzyme 2 receptors on olfactory mucosal cells and/or neuropilin- 1 on the olfactory epithelium ${ }^{6}$. Other potential routes of SARS-CoV-2 entry into the CNS could include the bloodstream, a breached blood-brain barrier and retrograde axonal transport ${ }^{3-5}$ (FIG. 1).

\section{4 the accumulating evidence on neurological sequelae in patients with COVID-19 is a cause for concern}

Unlike the oral cavity, the olfactory mucosa in the nose lacks lymphoid aggregates, and this feature could be exploited by SARS-CoV-2 to gain access to the CNS across the cribriform plate. En route to the CNS, SARS-CoV-2 seems to leave signatures in the form of traces of viral RNA and budding of virions from the cells in the olfactory epithelium, olfactory bulb and frontal lobe of the brain ${ }^{4,5,7}$. In addition, factors such as hypoxia, cerebral ischaemia and the cytokine storm might act together with the neurotrophic effects of SARS-CoV-2 to produce the neurological damage that is reported in COVID-19.

With the ongoing COVID-19 pandemic, clusters of patients have emerged with symptoms that continue beyond the acute phase of the disease - a condition commonly known as 'long COVID”. . A substantial proportion of these so-called 'long-haulers' have neurological manifestations, and there is an urgent need 


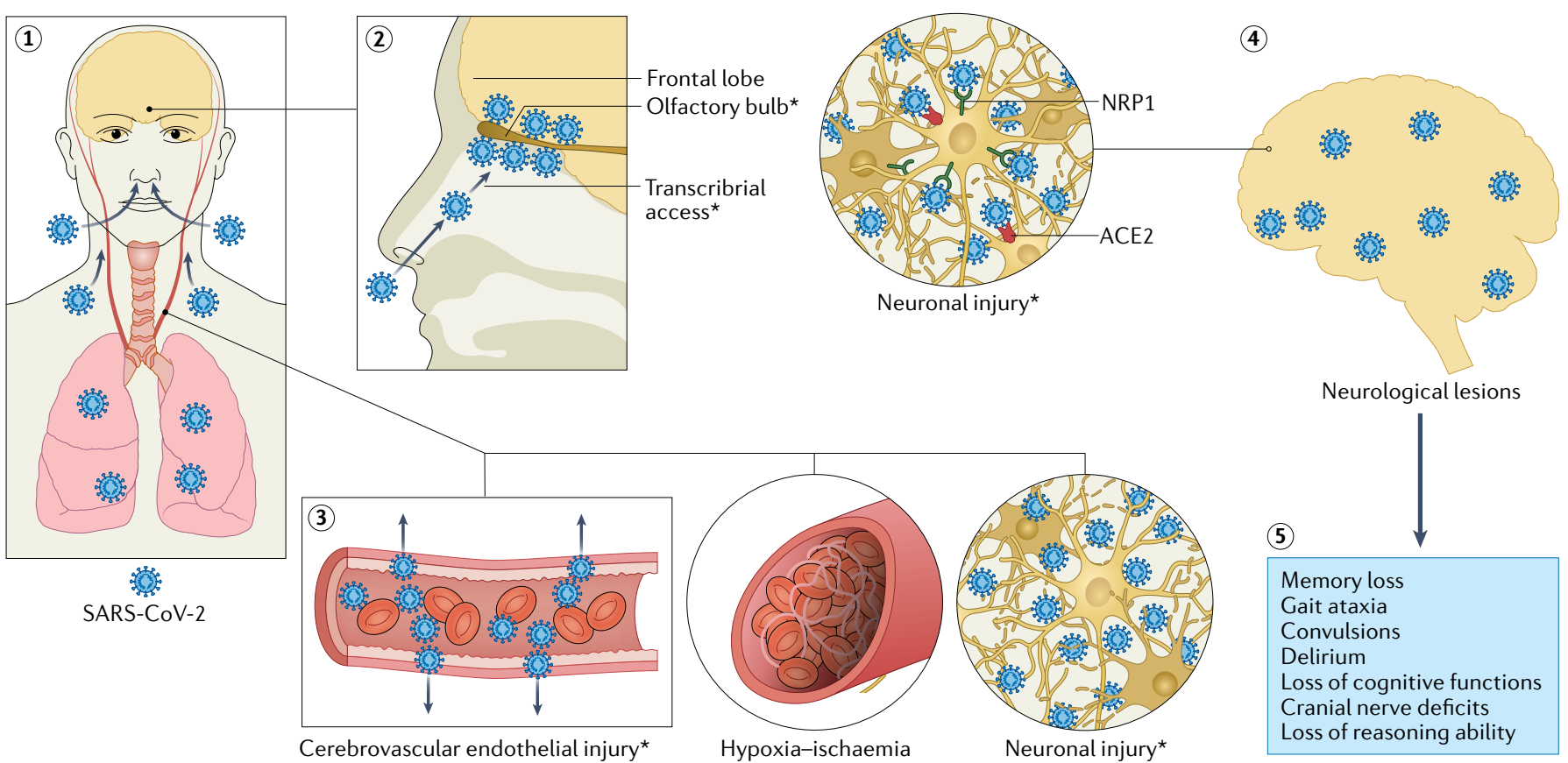

Fig. 1 | COVID-19-related neurological deficits. The figure illustrates proposed routes of the spread of SARS-CoV-2 across the CNS and the possible mechanisms involved in neuronal injury in COVID-19. Mechanisms indicated by asterisks were proposed on the basis of data that emerged from hospitals in Wuhan, China in the first two months of 2020 (REF. ${ }^{3}$ ). SARS-CoV-2 produces viral loads in the oral cavity and nose (1) that cause infection of the olfactory mucosa and olfactory bulb ${ }^{4}$ to reach the brain (2-4). Viral budding from neuronal cells in the frontal lobe has been documented ${ }^{7}$. Vascular mechanisms that can cause neuronal damage are shown (3). Brain involvement (4) is thought to elicit specific clinical features (5) that are indicative of CNS injury. ACE2, angiotensin-converting enzyme 2 receptor; NRP1, neuropilin-1. Adapted with permission from REF. $^{3}$. to address their health issues. A global consensus is needed on diagnostic approaches, management modalities and follow-up to ensure the well-being of long-haulers in general, and to address the neurological features of COVID-19 in particular'. Topical therapy with antiviral drugs and antibodies, administered via nebulizers and nasal sprays at the portal of entry of SARS-CoV-2, is expected to reduce viral loads and minimize the incidence of neurological complications ${ }^{10}$.

In conclusion, the accumulating evidence on neurological sequelae in patients with COVID-19 is a cause for concern. Without proper care and treatment, patients with these neurological manifestations might be permanently debilitated. Moreover, the continuation of neurological symptoms in long COVID could overwhelm already fractured health-care systems. Consequently, we urgently need to understand and respond to the covert yet potentially incapacitating neurological consequences of COVID-19 in the acute and chronic phases of the disease.

Abdul Mannan Baig iD

Department of Biological and Biomedical Sciences, Aga Khan University, Karachi, Pakistan. e-mail:abdul.mannan@aku.edu

https://doi.org/10.1038/s41582-021-00593-7

1. Misra, S. et al. Frequency of neurologic manifestations in COVID-19: a systematic review and meta-analysis. Neurology https://doi.org/10.1212/WNL 0000000000012930 (2021).

2. Sanchez, C. V., Theel, E., Binnicker, M., Toledano, M $\&$ McKeon, A. Autoimmune encephalitis postSARS-CoV-2 infection: case frequency, findings, and outcomes. Neurology https://doi.org/10.1212/ WNL.0000000000012931 (2021)

3. Baig, A. M., Khaleeq, A., Ali, U. \& Syeda, H. Evidence of the COVID-19 virus targeting the CNS: tissue distribution, host-virus interaction, and proposed neurotropic mechanisms. ACS Chem. Neurosci. 11. 995-998 (2020)
4. Meinhardt, J. et al. Olfactory transmucosa SARS-CoV-2 invasion as a port of central nervous system entry in individuals with COVID-19. Nat. Neurosci. 24, 168-175 (2021).

5. Burks, S. M., Rosas-Hernandez, H., Alejandro Ramirez-Lee, M., Cuevas, E. \& Talpos, J. C. Can SARS-CoV-2 infect the central nervous system via the olfactory bulb or the blood-brain barrier? Brain Behav. Immun. 95, 7-14 (2021).

6. Cantuti-Castelvetri, L et al Neuropilin-1 facilitates SARS-CoV-2 cell entry and infectivity. Science 370 , 856-860 (2020).

7. Paniz-Mondolfi, A. et al. Central nervous system involvement by severe acute respiratory syndrome coronavirus-2 (SARS-CoV-2). J. Med. Virol. 92, 699-702 (2020).

8. Nalbandian, A. et al. Post-acute COVID-19 syndrome Nat. Med. 27, 601-615 (2021).

9. Baig, A. M. Chronic long-COVID syndrome: a protracted COVID-19 illness with neurological dysfunctions. CNS Neurosci. Ther. https://doi.org/ 10.1111/cns. 13737 (2021).

10. Baig, A. M. Targeting neuroinvasion by SARS-CoV-2: emerging trends in drug and antibody delivery to combat COVID-19. ACS Chem. Neurosci. 12, 2555-2557 (2021).

Competing interests

The author declares no competing interests 